\title{
Hysteretic Model of a Vehicle Torsional Damper
}

\author{
Rong Zeng ${ }^{1,2}$, Ming Tu ${ }^{1,2}$ and Liwei Wang ${ }^{3, *}$ \\ ${ }^{1}$ College of Engineering, Huazhong Agricultural University, Wuhan 430070, China \\ ${ }^{2}$ Key Laboratory of Agricultural Equipment in Mid-lower Yangtze River, Ministry of Agriculture, Wuhan 430070, China
}

${ }^{3}$ Cummins East Asia Research \& Development Co., Ltd, Wuhan 430014, China

Received 21 May 2018; Accepted 21 August 2018

\begin{abstract}
Torsional dampers can significantly improve torsional vibrations of power transmission systems in vehicles that use internal combustion engines. Torsional stiffness and damping are key parameters that influence damping performance of torsional dampers. In this study, a hysteretic nonlinear model of a torsional damper was constructed to reveal torsional stiffness and damping behavior. The parameter estimation model of the transmitted torque of the torsional damper was established through experiments and parameter estimation. The torsional stiffness and damping characteristics of the torsional damper and the dynamic characteristics of the power transmission system were discussed based on the proposed model. The feasibility and accuracy of the proposed model were verified through experiments. Results demonstrate that torsional stiffness and damping have nonlinear relationships with rotational speed. Both increase with the rise of rotational speed. Torsional stiffness and damping of the torsional damper can help the power transmission system avoid idle resonance. However, they influence torsional vibration of the power transmission system under high-speed conditions slightly. The obtained conclusions are applicable to the analysis of damping performance of torsional dampers under any rotational speed, thereby offering certain theoretical references for design optimization of torsional damper parameters.
\end{abstract}

Keywords: Torsional damper, Torsional stiffness, Damping, Hysteresis nonlinearity, parameter estimation

\section{Introduction}

Vehicles such as automobile and tractors, which use internal combustion engines as power source, have torsional vibrations. The internal combustion engines are not only the power source of vehicles but also the excitation source of power transmission systems. During the operation of an internal combustion engine, the rotational speed of the crankshaft and the output torque of the engine have alternating characteristics, thereby generating torsional vibration of the crankshaft (hereinafter referred to as the torsional vibration) [1]. This phenomenon is attributed to the collaborative effect of reciprocating moments of inertia of the crankshaft and pistons, as well as the pressure torque of gas in the air cylinder. Torsional vibration is a key factor that influences ride comfort of vehicles and reliability of power transmission systems. In the automobile field, new energies are replacing traditional internal combustion engines, and the torsional vibration problem is not prominent anymore. However, in the agricultural field, tractors are being developed to be high-powered; therefore, internal combustion engines cannot be replaced in the predictable future, and the torsional vibration problem remains.

The common way to improve torsional vibration of internal combustion engines is to install a torsional damper at the output end of the crankshaft. A torsional damper is composed of elastic and damping elements. The former has relatively low torsional stiffness and can change the natural

\footnotetext{
*E-mail address: zengrong8802@163.com

ISSN: 1791-2377 @ 2018 Eastern Macedonia and Thrace Institute of Technology. All rights reserved. doi:10.25103/jestr.114.12
}

frequency of the power transmission system. Consequently, the resonance speed of the power transmission system can avoid the rotational speed of vehicles during normal driving, resulting in vibration isolation. Despite lubrication, the damping element of torsional dampers can consume vibration energy and attenuate torsional vibration amplitude, resulting in vibration absorption. Elastic elements of torsional dampers commonly use long arc springs. The lubricating grease, which is filled in the spring channels, offers lubrication and damping effects.

Torsional stiffness and damping are key parameters that influence damping performance of torsional dampers. Because of the collaborative actions of the elastic and damping elements, torsional dampers transmit the engine torque, resulting in the nonlinear characteristics of the transmitted torque. In early designs of the arc springs, the linear spring theory was adopted to obtain the relationship between the transmitted torques and torsion angles of the arc springs, thereby establishing calculation models of torsional stiffness of the torsional dampers [2-4]. Since the influence of damping was neglected, these models were linear and have poor accuracy in analyzing torsional vibration characteristics of power transmission systems. With the continuous improvement of experimental methods, progress has been achieved in research on influences of damping on dynamic behaviors of torsional dampers. An experiment on the dynamic responses of a torsional damper reflected that the natural frequency of the damper would increase with rise of rotational speed [5]. Experiments on the behavior of torsional dampers revealed that the dampers would consume energies in the reciprocating movement of the arc springs, resulting in different torsional stiffness in compression and 
decompression of the arc springs [6, 7]. These experimental results demonstrated that torsional dampers presented nonlinear characteristics. The corresponding mechanism was elaborated in the follow-up theoretical studies [8, 9]. Arc springs, which possess recovery characteristics, generate elastic torque during the working process. Meanwhile, the damping torque generated by the damping element is divergent, and system energy is consumed. When excitation torque is reciprocating, torsional dampers characterize hysteresis nonlinearity [8]. In addition, the arc springs must be influenced by the centrifugal force during the reciprocating movement because torsional dampers act on power transmission systems. The centrifugal force changes with engine speed. Hence, transmitted torque of torsional dampers is correlated with rotational speed [9]. Although studies on nonlinear characteristics of torsional dampers are many [5-9], no general model can describe torsional dampers due to the close relationship of damping with rotational speed and temperature and the dynamic characteristics of the lubricating state [10]. Certain differences between the analysis results and actual situations existed because the analyses on torsional vibration characteristics of the power transmission systems were implemented under a specific rotational speed. Therefore, establishing a general model of the transmitted torque of the torsional damper and acquiring the dynamic characteristics of the torsional stiffness and damping are problems that must be solved.

\section{State of art}

Many scholars have conducted abundant studies on torsional dampers for vehicles. They focused on the designs of arc springs, constructions of the torsional vibration models of power transmission systems, analysis of vibration damping performance, as well as studies on various experimental methods.

Shi Wenku et al. [11] designed the segmented nonlinear torsional stiffness for a torsional damper and applied the torsional stiffness into the power transmission system with multi-degree of freedom (DOF) to analyze damping performance. To adapt to different working conditions, the arc springs were innovatively designed into three-level nonlinear torsional stiffness, during which influences of rotational speed on the torsional stiffness and changes of frictional damping were overlooked. Lamkaned et al. [12] analyzed the elastic characteristics of a torsional damper and acquired the torsional stiffness. However, frictional factors were neglected, and the gained torsional stiffness was the result in the static state. Using the linear elastic design theory, Lv Zhenhua et al. [13] established the linear design model of the arc spring by analyzing torsional vibration characteristics of the power transmission system. This model deduced the relationship between the transmitted torque and torsion angle of the arc spring. Based on the lamped mass model with multi-DOF of the power transmission system, Chen Lei et al. [14] studied the relationship among the torsional stiffness, moment of inertia, and the natural frequency of the torsional damper. On this basis, the design methods for the moment of inertia and the idle torsional stiffness of the torsional damper were constructed. However, neither modeling nor deduction of the design methods had considered the effects of frictional damping. Shelke et al. [15] introduced the vibration attenuation principle of a torsional damper, but did not discuss the influences of damping effects on the vibration damping performance. Swapnil et al. [16] introduced the design principle of the moment of inertia of a torsional damper and elaborated the frictional behaviors of the damper. Nevertheless, they did not construct a specific friction model. Yan Minggang et al. [17] accomplished the optimized design of the parameters of a torsional damper for a coaxial and series hybrid-powered bus. Through response sensitivity analysis, they determined major factors that influenced the torsional vibration level of the power transmission system. The maximum torsion angle under full working conditions was used as the objective function, and the torsional stiffness of the torsional damper was optimized. However, the optimization process neglected influences of damping factors on torsional stiffness. Xiang Changle et al. [18] proposed a semi-active controlling torsional damper based on the positive and negative stiffness in series and deduced the corresponding mathematical expression of the stiffness. On this basis, they analyzed the elastic characteristics of the damper, but neglected the factors concerning frictional damping. Philipp Mall et al. [19] proposed an automatic parameter optimization design method for torsional dampers based on Augmented Lagrangian Particle Swarm Optimization method. Based on the lightweight design, the spatial layout and the moment of inertia of the torsional damper were optimized. Jong-Yun Yoon et al. [20] studied influences of the torsional damper of multilevel stiffness clutch on the rattle of manual transmission. The linear and nonlinear torsional vibration models of the power transmission system were constructed through modal analysis, and then, the rattle phenomenon was simulated. Shu Gequn et al. [21] analyzed the influences of damping ratio of the torsional damper on the amplitude amplification coefficient of torsional vibration response of the power transmission system. Based on analysis results, they also proposed the idea of variable damping for torsional damper. Tejashri et al. [22] analyzed and studied performances of the internal combustion engine, which used the multilevel spring torsional damper; however, the dynamic characteristics of the torsional damper were overlooked in their study. Shimizu et al. [23] discussed the performance of a spring torsional damper in low-frequency vibration damping during the accelerating process of vehicles. Parameters in the constructed model were undetermined, and the model was linear. Zhen Guangze et al. [24] studied the influences of a torsional damper on the Noise, Vibration and Harshness (NVH) performance of vehicles. However, they adopted fixed values of torsional stiffness and damping of the torsional damper. Tejashri et al. [25] compared the performances of a two-stroke engine with and without spring torsional damper. The study involved an experimental study on the engine performance, but the theoretical analysis of influences of the dynamical parameters on the engine performance was not conducted. Liu Shengtian [26] analyzed the performance of a torsional damper in controlling vibration and noise of vehicles under idle conditions. He analyzed the inherent torsional vibration characteristics by establishing a lamped mass model, but damping factors were neglected, and the torsional stiffness in this model was a constant. Song Liquan et al. [27] initially introduced friction into a circumferential short-spring dual mass flywheel (DMF) of vehicles and deduced the relationship between the transmitted torque and torsion angle, obtaining the mechanical model of the torque characteristic. $\mathrm{Li}$ Jie et al. [28] developed a test bench to evaluate performances of a torsional damper for vehicles based on virtual instrument technology. The control system of the test 
bench simulated the torsional vibration generated by engines and realized adjustment of rotational speed, frequency, and amplitude of torsional vibration excitation through an inverter and a variable frequency motor. Jiang Zhengfeng et al. [29] suggested a method to realize excitation by adjusting rotational speed and loads and by using a hydraulic cylinder.

Some studies have achieved the mathematical model of the transmitted torque of torsional dampers, and some studies have applied the models into power transmission systems. However, the models were mainly linear, and the torsional stiffness deduced by the models was constant because dynamic characteristics of torsional stiffness and damping effect were neglected. Studies concerning torsional stiffness and damping nonlinearity of the torsional damper and their influences on torsional characteristics of a vehicle are rare. This study proposes a hysteretic nonlinear model of transmitted torque of a vehicle torsional damper through experiments and parameter estimation. Aiming to disclose the torsional characteristics of the power transmission system under full speed conditions, nonlinearities of torsional stiffness and damping, and the dynamic characteristics of the power transmission system are analyzed. Results of this study can provide theoretical references for optimal design of torsional stiffness and damping parameters of the torsional damper.

The remainder of this study is organized as follows. Section 3 accomplishes the torsion test of the torsional damper and establishes the hysteretic nonlinear transmitted torque model. Section 4 analyzes torsional stiffness and damping characteristics of the torsional damper and the dynamic characteristics of the power transmission system. Section 5 summarizes the conclusions.

\section{Methodology}

\subsection{Torsion test}

This study introduces a torsional damper with long-arc springs, as shown in Fig.1. The torsional damper is installed between the engine and transmission. The movements of the arc springs are constrained by the spring channels. The arc spring can be compressed to transmit engine torque. To relieve abrasion of the arc springs, the spring channels are filled with lubricating grease, which offers lubrication and damping effect.

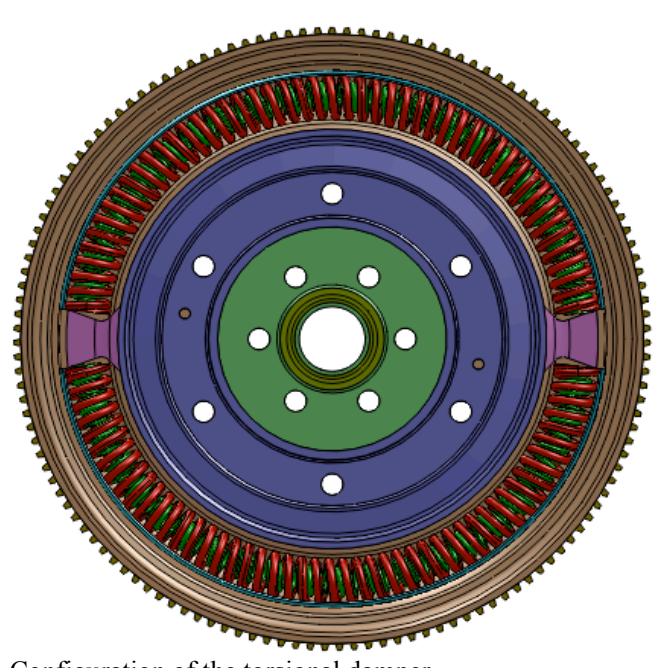

Fig. 1. Configuration of the torsional damper

A torsion test is performed to show the relationship between the transmitted torque and torsion angle of the torsional damper. The torsion test is accomplished via a torsional behavior test system, which is composed of the control unit, test bench, and data acquisition (DAQ) and analysis unit, as shown in Fig.2. The excitation controller in the control unit is used to regulate the converter motor to generate torsional vibration to simulate the actual working condition of vehicles. The load controller regulates a dynamometer to generate load torque, simulating load conditions of vehicles under different driving conditions. The test bench, as shown in Fig.3, is composed of the converter motor, the torsional damper, the dynamometer, and torque-speed sensors. The torque-speed sensors can detect the torque and rotational speeds at the front-end input and rear-end output of the torsional damper. Signals are acquired and processed by the DAQ and analysis unit, aiming to obtain the transmitted torque and torsion angle of the damper. In the test process, the load controller controls the dynamometer to apply loads. Initially, the load torque is continuously increased from 0 to $370 \mathrm{~N} \cdot \mathrm{m}$ and then decreased to 0 . Afterward, the load torque is subsequently increased from 0 to $370 \mathrm{~N} \cdot \mathrm{m}$ in the opposite direction and finally decreased to 0 . The transducer controls the converter motor to rotate under different rotational speeds. The relationships between the transmitted torque and the torsion angles under different rotational speeds $(100,1000,2000$, and $3000 \mathrm{r} / \mathrm{min}$ ) are recorded in Fig 4.

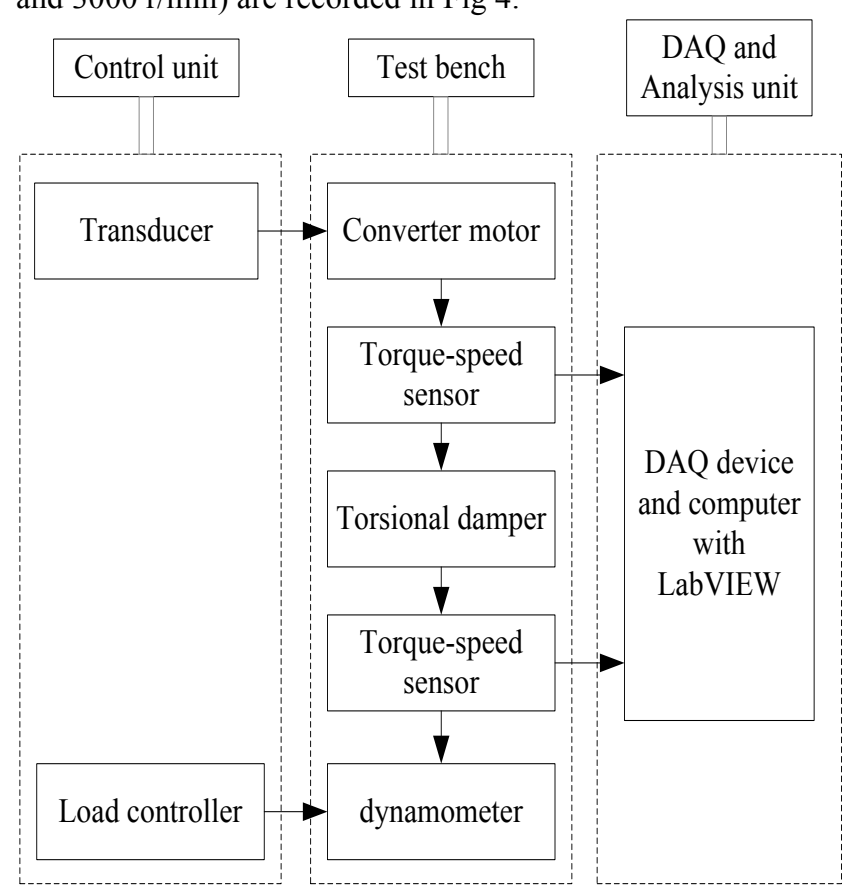

Fig. 2. Test system for torsional behavior of torsional damper

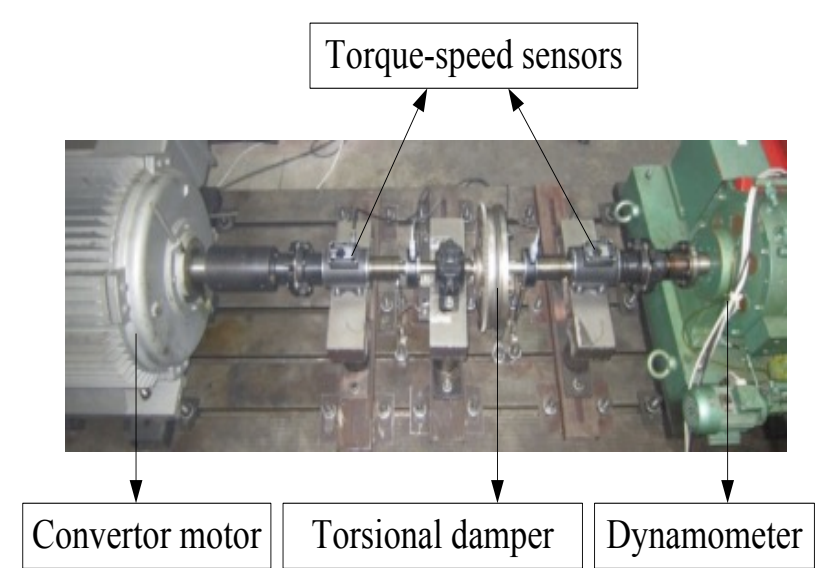

Fig. 3. Test bench 


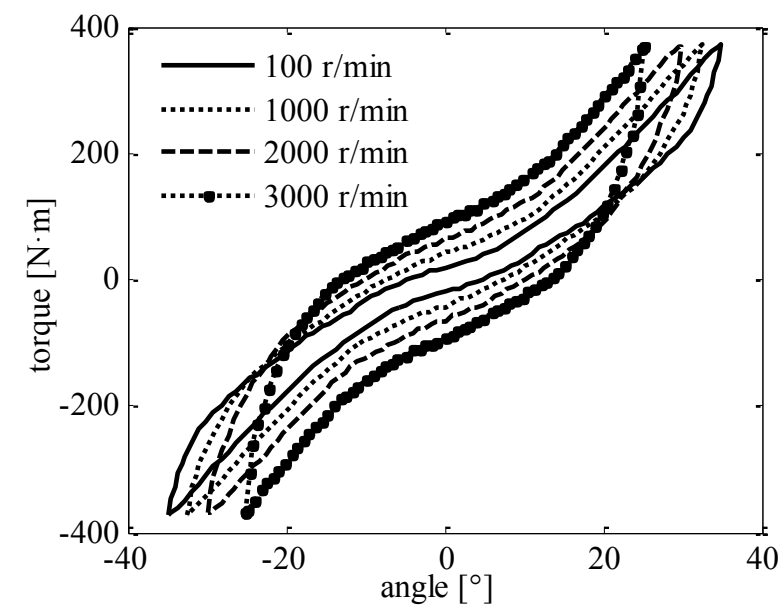

Fig. 4. Experimental torque-angle curves

\subsection{Establishment of the hysteretic nonlinear model}

The torsional damper transmits engine torque under the collaborative effect of the elastic and damping elements. The transmitted torque is the superposition of the elastic and damping torque. Experimental results in Section 3.1 show that the transmitted torque is nonlinear. Thus, it can be decomposed into nonlinear elastic and nonlinear damping torque. The nonlinear elastic torque is generally related to torsion angle, and it can be expressed as a polynomial function of torsion angle

$$
T_{s}(\theta)=\sum_{i=0}^{(m+1) / 2} k_{2 i+1} \theta^{2 i+1}
$$

Where $\theta$ denotes the deformation angle of the arc springs, i.e., the torsion angle of the torsional damper. Viewed from the decomposition of the hysteretic curves, the polynomial stiffness can meet the requirements of estimation accuracy and $m=3$. It can be further expanded as

$$
T_{s}(\theta)=\sum_{i=0}^{(m+1) / 2} k_{2 i+1} \theta^{2 i+1}=k_{1} \theta+k_{3} \theta^{3}+k_{5} \theta^{5}
$$

Where $k_{i}$ denotes the dynamic stiffness coefficient.

The nonlinear damping torque can be described as

$T_{c}(\theta, \dot{\theta})=\sum_{i=0}^{(m-1) / 2} c_{2 i} \theta^{2 i} \operatorname{sign}(\dot{\theta})$

During the working process of the torsional damper, the arc springs make reciprocating movements under the effect of fluctuating torque. The lubricating grease and spring shells generate damping torque to the arc springs. The damping torque is related to the materials of the spring shells and arc springs, temperature of the lubricating grease, the deformation speed, and the area and roughness of the contact surface. It cannot be described by single coulomb or viscous damping and is generally the comprehensive reflection of multiple damping components. To describe the damping components of the torsional damper accurately, damping factor is introduced to the damping torque model. With clear physical meaning, the damping factor can be used to describe multiple damping components. Therefore, the nonlinear damping torque can be rewritten as

$$
T_{c}(\theta, \dot{\theta})=c|\dot{\theta}|^{n} \operatorname{sign}(\dot{\theta})
$$

Where $c$ represents the coefficient of the damping components, and $n$ denotes the damping factor which reflects sensitivities of damping torque to changes. When $n=0$, the system represents coulomb damping. When $n=1$, the damping of the system is viscous. When $0<n<1$, the system contains both coulomb and viscous damping. When $n>1$, the system manifests highorder damping.

In summary, the hysteretic nonlinear torque model of the torsional damper can be expressed as

$$
\begin{aligned}
& T(\theta, \dot{\theta})=T_{k}(\theta)+T_{c}(\theta, \dot{\theta}) \\
& =k_{1} \theta+k_{3} \theta^{3}+k_{5} \theta^{5}+c|\dot{\theta}|^{n} \operatorname{sign}(\dot{\theta})
\end{aligned}
$$

Where $k_{1}, k_{3}, k_{5}, c$ and $n$ are parameters to be determined.

\subsection{Parameter estimation}

The damping torque parameters in Equation (5) are not independent; thus, the model parameters cannot be estimated by the least square method directly. In this study, parameter estimation can be accomplished in two steps, as follows:

Step 1: Given a value of $n$, other parameters are estimated using the least square method.

The torsion angle sequence $\left\{\theta_{i}\right\}$ and the torque sequence $\left\{T_{i}\right\}$ can be gained through the torsion test in Section 3.1. Under this circumstance, the torsion angle and torque sequences are results after re-sampling with an equivalent time interval, which implies that the numbers of data points of torsion angle and torque sequence are equal. Here, $i$ represents the number of corresponding sampling points, and $i=1,2, \cdots, N$, where $N$ is the maximum number of the sampling points, that is, the signal lengths of torque and torsion angle. If the sampling interval is $\Delta t, \theta_{i}=\theta(i \cdot \Delta t)$. The torsion speed can be obtained from discrete differential: $\dot{\theta}_{i}=\left(\theta_{i+1}-\theta_{i}\right) / \Delta t$, and the rotational speed sequence $\left\{\theta_{i}\right\}$ can be obtained, where the sequence length is $N-1$.

Step 2: Parameters are estimated by the LevenbergMarquardt optimization algorithm [30]. In this step, the parameters (including the given $n$ ) estimated in Step 1 are used as an initial iteration vector. Levenberg-Marquardt algorithm has higher robustness and efficiency than the widely used Gauss-Newton algorithm and can overcome the slow convergence of Gauss-Newton algorithm. Therefore, the parameter estimation problem can be converted into a optimization problem. The algorithm calculates the parameter sequence corresponding to the minimum of residual equation, and the residual equation can be calculated by

$$
R(t)=k_{1} \theta+k_{3} \theta^{3}+k_{5} \theta^{5}+c|\dot{\theta}|^{n} \operatorname{sign}(\dot{\theta})-T
$$

Where $T$ is the transmitted torque measured by actual test. 
The error equation is constructed by the residual equation and it can be expressed as

$$
e(t)=\sum_{k=1}^{M}[R(t)]^{2}=\sum_{k=1}^{M} R_{k}^{2}
$$

Let $\{\varphi\}=\left\{\varphi_{1}, \varphi_{2}, \varphi_{3}, \varphi_{4}, \varphi_{5}\right\}^{T}=\left\{k_{1}, k_{3}, k_{5}, c, n\right\}^{T}$. Calculate the partial derivative of $\varphi_{i}(i=1,2, \cdots, 5)$ using Equation (7). The resultant equation is:

$q_{i}=\frac{\partial e(t)}{\partial \varphi_{i}}=2 \sum_{k=1}^{M} R_{k} \frac{\partial R_{k}}{\partial \varphi_{i}}$

The $\partial R_{k} / \partial \varphi_{i}$ forms the elements of the Jacobian matrix:

$$
\left\{\begin{array}{l}
\frac{\partial R_{k}}{\partial \varphi_{1}}=\theta \\
\frac{\partial R_{k}}{\partial \varphi_{2}}=\theta^{3} \\
\frac{\partial R_{k}}{\partial \varphi_{3}}=\theta^{5} \\
\frac{\partial R_{k}}{\partial \varphi_{4}}=|\dot{\theta}|^{n} \operatorname{sign}(\dot{\theta}) \\
\frac{\partial R_{k}}{\partial \varphi_{5}}=c|\dot{\theta}|^{n} \operatorname{sign}(\dot{\theta}) \cdot \ln (|\dot{\theta}|)
\end{array}\right.
$$

The Hessian matrix can be established, and the matrix elements are:

$$
H_{i j}=\frac{\partial^{2} e(t)}{\partial \varphi_{i} \partial \varphi_{j}}=2 \sum_{k=1}^{M} R_{k} \frac{\partial^{2} R_{k}}{\partial \varphi_{i} \partial \varphi_{j}}+2 \sum_{k=1}^{M} \frac{\partial R_{k}}{\partial \varphi_{i}} \cdot \frac{\partial R_{k}}{\partial \varphi_{j}}
$$

The first term at the right side of the equation contains $R_{k}$ and $R_{k}$ is close to zero when the parameter estimation is very close to the actual situation. Thus, the first term at the right side of the equation can be neglected, and the second term is maintained. The iteration equation [30] of the Levenberg-Marquardt optimization algorithm can be expressed as

$$
\varphi_{i+1}=\varphi_{i}-\left[H\left(\varphi_{i}\right)+\lambda_{i} I\right]^{-1} q\left(\varphi_{i}\right)
$$

Where $\lambda_{i}$ is the Levenberg-Marquardt parameter, which is used to control the step length and direction of the next iteration step. The iteration steps are as follows.

(1) Parameter sequence, which is estimated in the first step, is used as the initial iteration value $\{\varphi\}_{1}$ to calculate the error $e_{1}(t)$ and the elements of Hessian matrix. An appropriate $\lambda_{i}$ is selected, and the next step of iteration vector $\{\varphi\}_{2}$ can be calculated according to Equation (11). The new error $e_{2}(t)$ can be calculated from $\{\varphi\}_{2}$. If $e_{2}(t)<e_{1}(t)$, it determines whether $\{\varphi\}_{2}$ is usable, or the iteration fails. Under this circumstance, $\lambda_{i}$ shall be added to recalculate until the acceptable $\{\varphi\}_{2}$ is obtained.

(2) The initial iteration value in the second step $\{\varphi\}_{2}$ is determined by the first step. Under this circumstance, the error is $e_{2}(t)$ and Step (1) is repeated. Continue to calculate the acceptable initial value for next iteration.

(3) Repeating the above steps, the terminal condition can be met until the error is small enough. Under this circumstance, the iteration parameters are the final parameter estimation results and can be used in the transmitted torque model of the torsional damper.

Under different rotational speeds, the above parameter estimation steps are accomplished by using the test data in section 3.1. Results of parameter estimation under different rotational speeds are shown in Table 1.

Table 1. Results of parameter estimation under different rotational speeds

\begin{tabular}{c|c|c|c|c|c}
\hline $\begin{array}{c}v \\
(\mathrm{r} / \mathrm{min})\end{array}$ & $k_{1}$ & $\begin{array}{c}k_{3} \\
\left(\times 10^{-3}\right)\end{array}$ & $\begin{array}{c}k_{5} \\
\left(\times 10^{-6}\right)\end{array}$ & $c$ & $\begin{array}{c}n \\
\left(\times 10^{-1}\right)\end{array}$ \\
\hline 500 & 5.592 & 4.182 & 0.373 & 13.572 & 1.781 \\
750 & 5.603 & 4.497 & 0.424 & 11.598 & 2.162 \\
1000 & 5.594 & 4.716 & 0.511 & 9.949 & 2.449 \\
1250 & 5.606 & 4.983 & 0.639 & 8.855 & 2.678 \\
1500 & 5.596 & 5.213 & 0.867 & 7.291 & 3.122 \\
1750 & 5.587 & 5.538 & 1.224 & 6.467 & 3.349 \\
2000 & 5.609 & 5.812 & 1.797 & 5.451 & 3.709 \\
2250 & 5.593 & 6.138 & 2.716 & 4.524 & 4.101 \\
2500 & 5.592 & 6.364 & 4.223 & 4.081 & 4.273 \\
2750 & 5.606 & 6.671 & 6.628 & 3.385 & 4.628 \\
3000 & 5.598 & 6.887 & 10.565 & 2.818 & 4.989 \\
\hline
\end{tabular}

Fig.5 compares the results of the experiments and estimation under different rotational speeds (1000, 2000 and $3000 \mathrm{r} / \mathrm{min}$ ). The results of estimation are close to that of experiment and the error distributions of transmitted torque under different rotational speeds $(1000,2000$ and $3000 \mathrm{r} / \mathrm{min})$ are shown in Fig.6. The standard deviations of the results of parameter are 4.34, 3.75, and 4.91, respectively. All errors are in allowable range and can meet the parameter estimation requirement, which verifies the reliability of the parameter estimation.

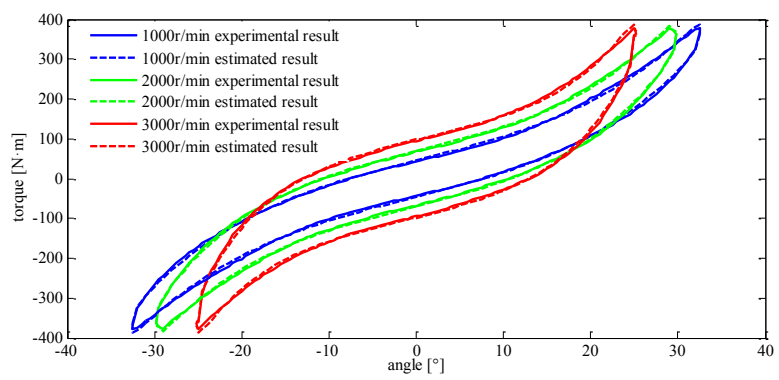

Fig. 5. Comparison of results of parameter estimation and experiment

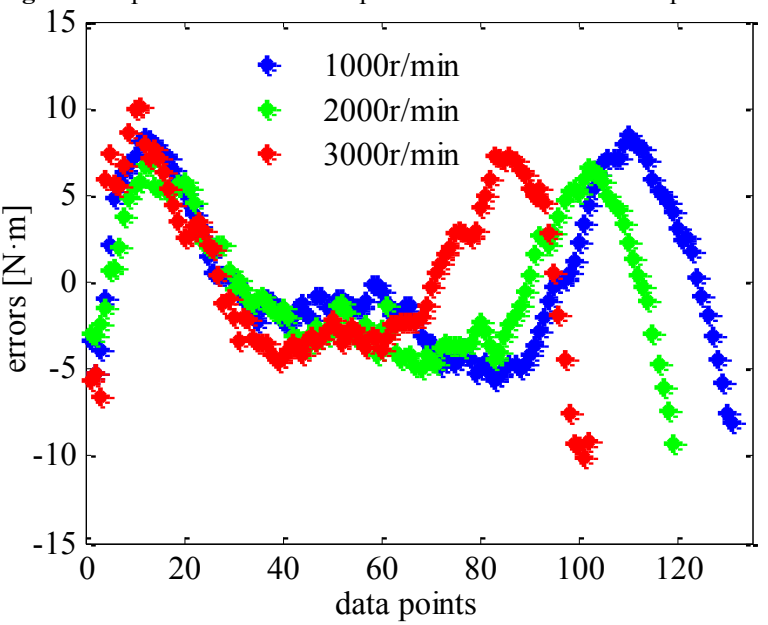

Fig.6. Error distributions 
3.4 Simulation model under full rotational speed

Experimental results in Section 3.1 reveal that the hysteretic nonlinear characteristics of the torsional damper change with rotational speed. Therefore, parameters in the proposed model must have certain functional relationships with rotational speed. To determine the relationships, the curves describing the variations of the estimated parameters and the rotational speed $v$ are plotted according to estimation results in Table 1, as shown in Figs.7 to 11. The formulas of each estimation parameter with respect to rotational speed are obtained through regression analysis, and the results are listed in Table 2.

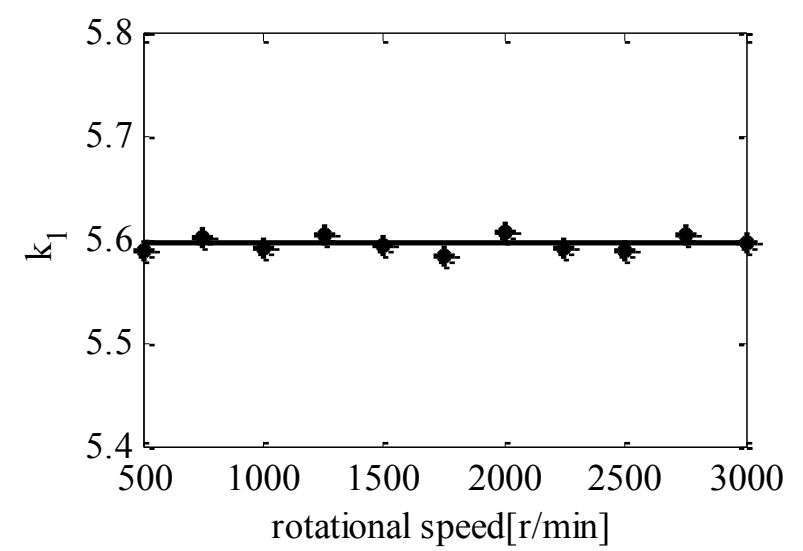

Fig. 7. Fitting results of $k_{1}$

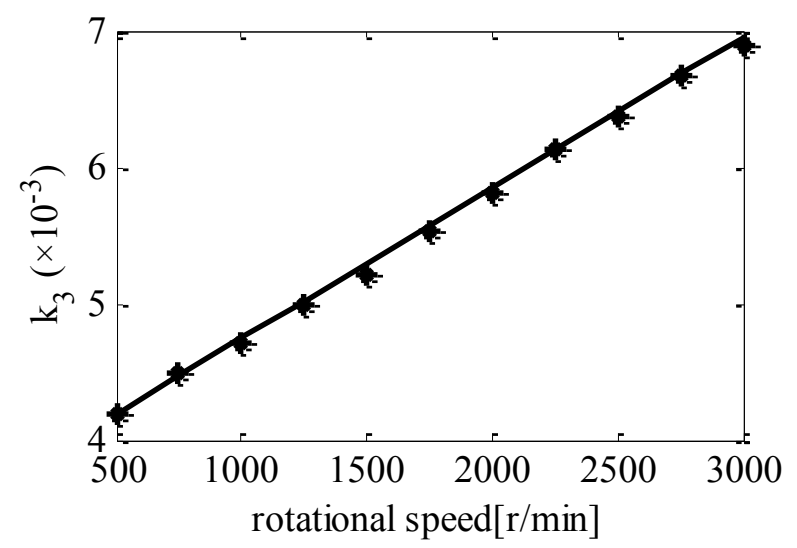

Fig. 8. Fitting results of $k_{3}$

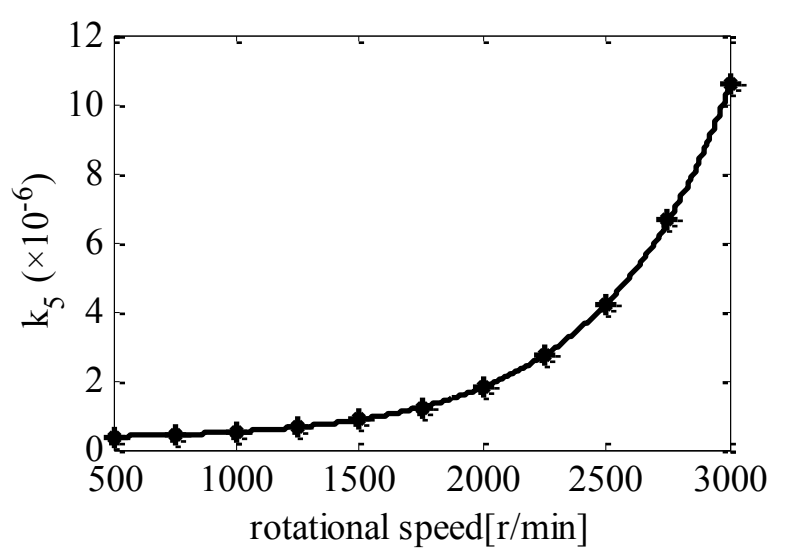

Fig. 9. Fitting results of $k_{5}$

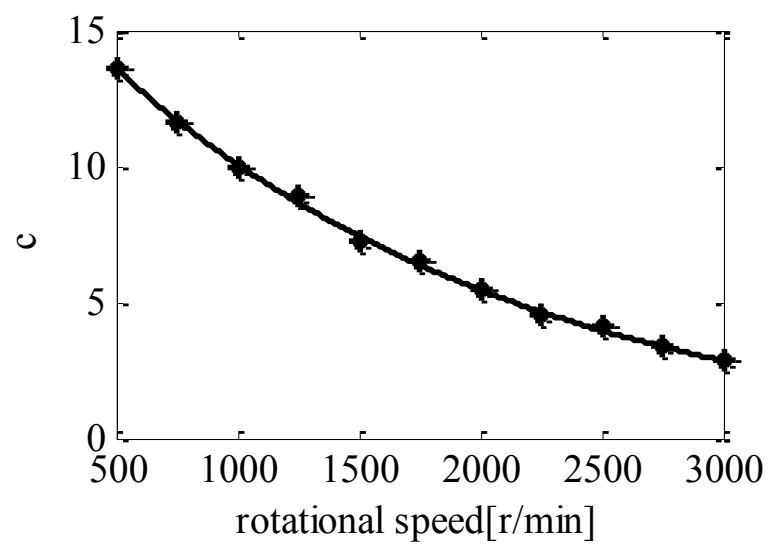

Fig. 10. Fitting results of $c$

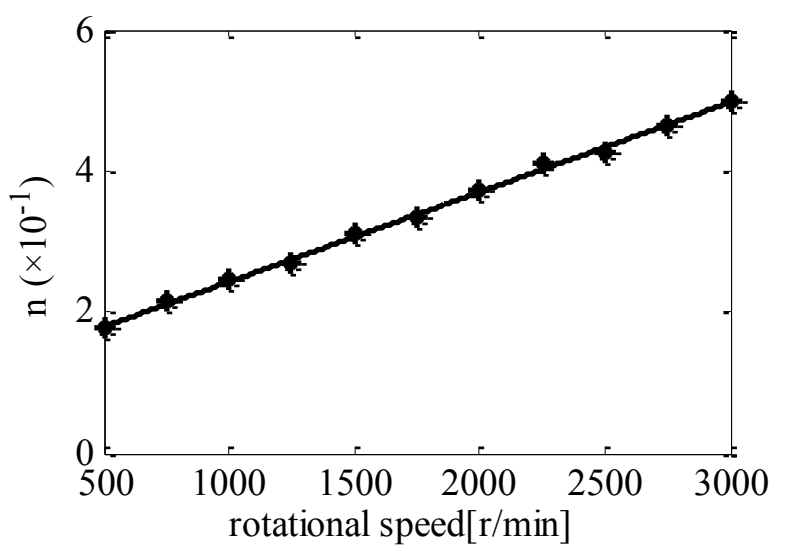

Fig.11. Fitting results of $n$

Table 2. Fitting results of estimated parameters

\begin{tabular}{c|c|c|c}
\hline Parameters & Fitting results & $\begin{array}{c}\text { Correlation } \\
\text { coefficient }\end{array}$ & Standard deviation \\
\hline$k_{1}$ & 5.598 & None & 0.00682 \\
$k_{3}$ & $k_{3}=(0.001109 v+3.631) \times 10^{-3}$ & 0.9994 & 0.0415 \\
$k_{5}$ & $k_{5}=\left(0.0322 e^{(0.00192 v)}+0.298\right) \times 10^{-6}$ & 0.9999 & 0.00196 \\
$c$ & $c=19.941 \cdot(60 / v)^{(0.0001272 v+0.1149)}$ & 0.9991 & 0.1128 \\
$n$ & $n=(0.001272 v+1.149) \times 10^{-1}$ & 0.9998 & 0.0048 \\
\hline
\end{tabular}

The fitting expressions of all estimated parameters in Table 2 are substituted into Equation (5) and then the transmitted torque model of the torsional damper under full rotational speed can be obtained. According to the established model, the relationships between the transmitted torque and torsion angle under different rotational speeds can be simulated, as shown in Fig.12. The results of simulation demonstrate that the transmitted torque is positively related to torsion angles, and the torque-angle curve becomes sharper as rotational speed increases. The enclosed areas of the curves increase as the rise of rotational speed. The results of simulation and experiment under 
rotational speeds of 1000,2000 , and $3000 \mathrm{r} / \mathrm{min}$ are compared in Fig.13. The comparison shows that the results of simulation are close to that of experiment, indicating that the constructed transmitted torque model based on the fitting results is feasible.

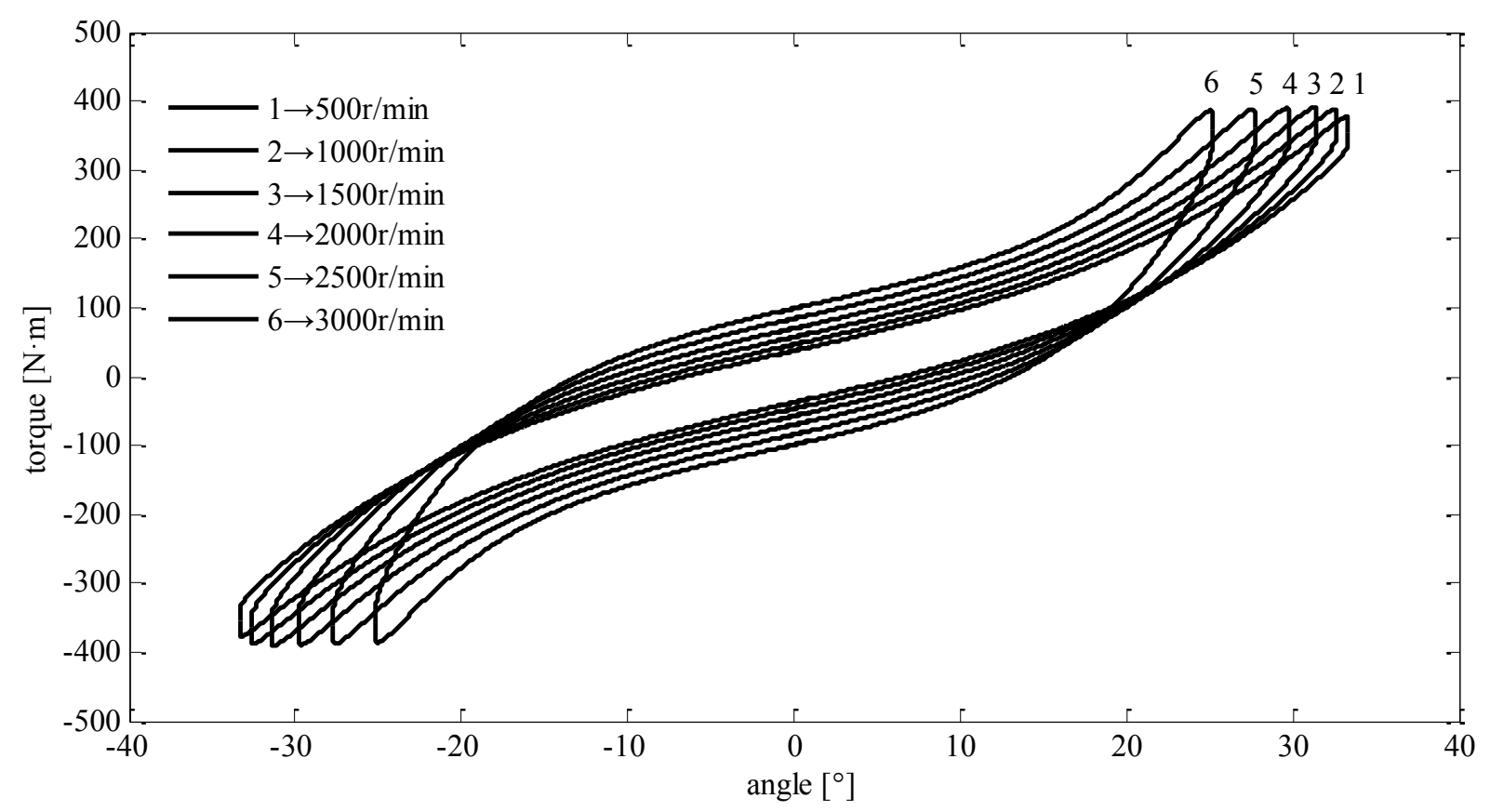

Fig. 12. Simulation torque-angle curves

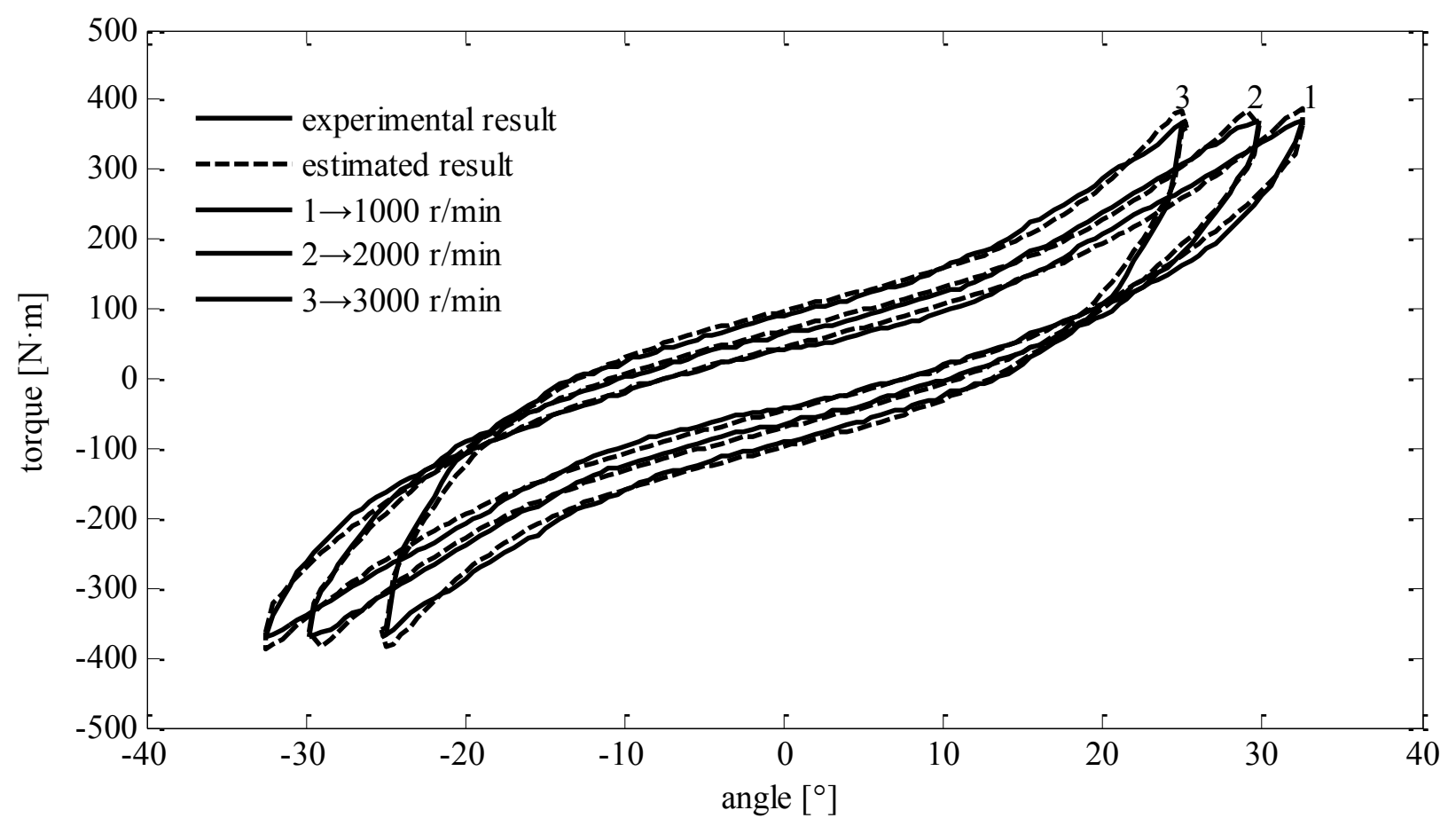

Fig.13. Comparison of results of simulation and experiment

\section{Result analysis and discussion}

\subsection{Analysis of torsional stiffness and damping}

The nonlinear elastic torque of the torsional damper can be acquired according to the previous three parameters fitted in Section 3.3, whereas the nonlinear damping torque can be obtained from the last two parameters. Relationships among the elastic torque, damping torque, and torsion angle are plotted, as shown in Fig.14 and Fig.15. Fig.14 shows that nonlinearity exists in the elastic torque. With the rise of rotational speed, the curve slope increases gradually, which reflects the positive relationship between the equivalent torsional stiffness and rotational speed. Fig. 15 indicates that the damping torque characterizes nonlinearity, and the damping torque and torsion angle form hysteretic loop. The area of the hysteretic loop enlarges with the rotational speed and torsion angle, which reflects that the energy consumed by damping increases with the torsion angle and rotational speed. Therefore, the equivalent damping of the torsional damper increases with the rise of rotational speed and torsion angle. 


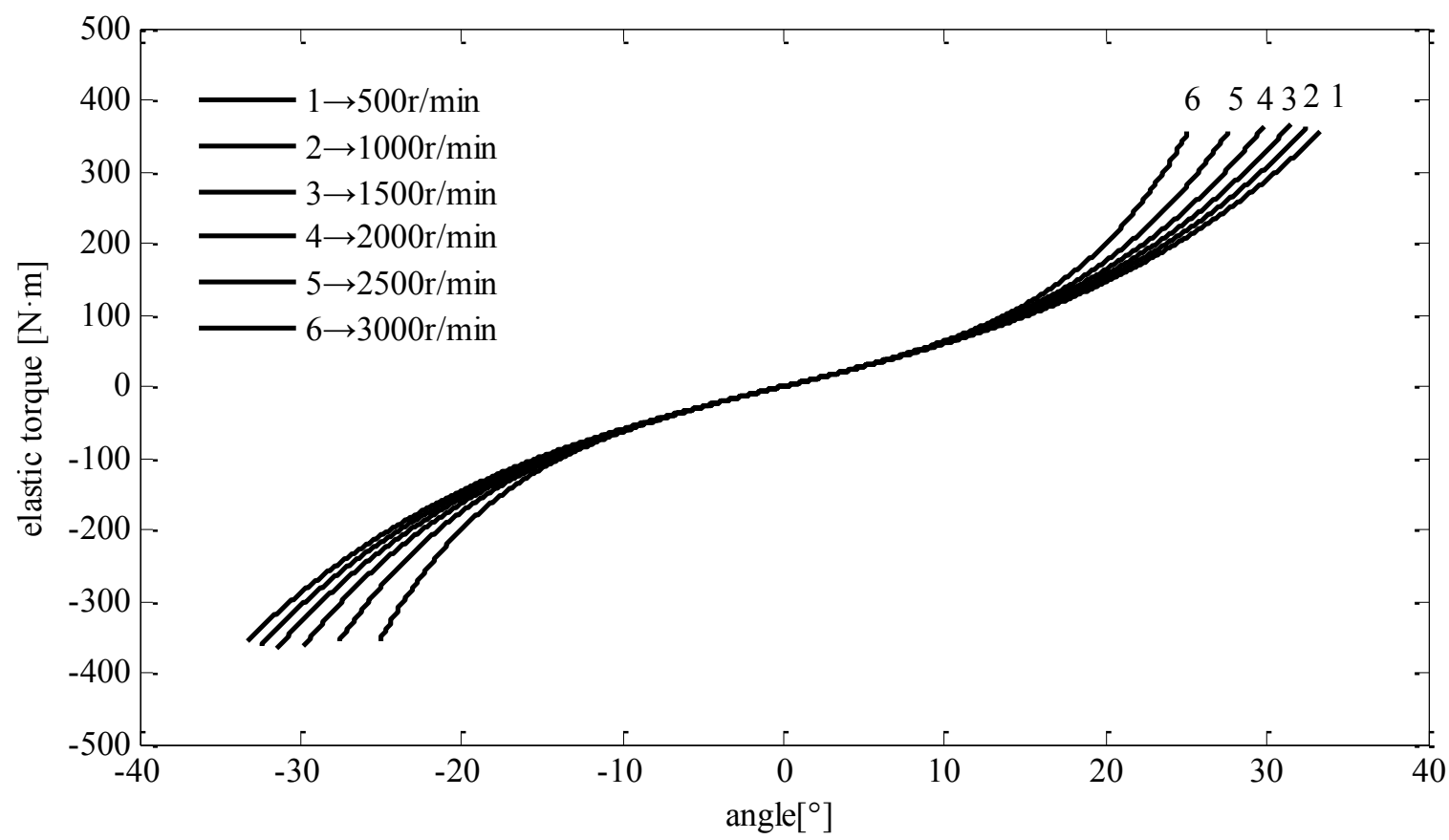

Fig. 14. Elastic torque-angle curves

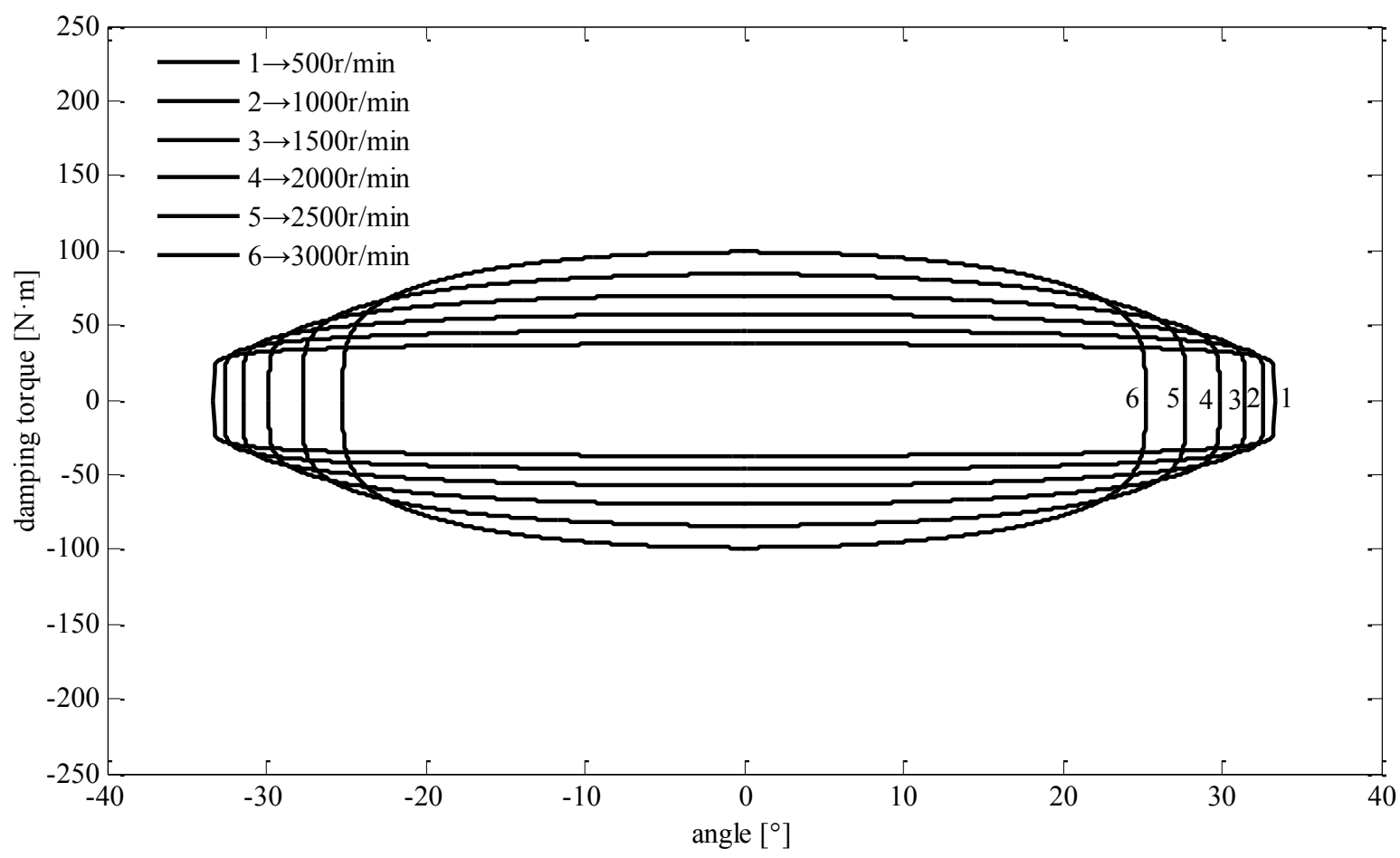

Fig. 15. Damping torque-angle curves

\subsection{Analysis of dynamic characteristics}

The power transmission system is equivalent to a $2-\mathrm{DOF}$ torsional vibration system. The torsional stiffness and damping of the system are equal to that of the torsional damper. The system response when torsional stiffness increases with rotational speed is predicted, as shown in Fig.16. Fig. 16 shows that the $1^{\text {st }}$-order resonant rotational speed of the power transmission system is approximately equal to $580 \mathrm{r} / \mathrm{min}$, which is lower than the idle speed $(800$ $\mathrm{r} / \mathrm{min}$ ). During start, the vehicle runs through the resonance region quickly because the torsional damper has low torsional stiffness at low speed, resulting in the low resonant rotational speed of the system. When the power transmission system runs through the resonance region, the torsional vibration amplitude at the input end of the transmission declines quickly and becomes smaller than that at the output end of the engine. During all driving conditions, the torsional vibration amplitude at the input end of the transmission is smaller than that at the engine's output end. The torsional stiffness of the torsional damper is positively related to rotational speed. Since the vibration amplitude of the system is small in the high-speed region, and the torsional vibration amplitude at the input end of the transmission is smaller than that at the output end of the engine (Fig.17), the torsional stiffness weakly influences the damping performance of the torsional damper. Changes in 
the system response with damping are analyzed through simulation, as shown in Fig.18. To realize damping effects, damping in the torsional damper not only lubricates the arc springs but also consumes the vibration energy of the power transmission system. The power transmission system has large torsional vibration amplitude in the resonance region, and the torsional damper can consume the torsional vibration energy by acquiring large damping. Thus, the torsional vibration amplitude decreases with the increase of damping. As rotational speed continuously increases, the rise of the damping becomes insignificant to the torsional vibration amplitude, which reflects that large damping may slightly influence the torsional vibration characteristics in the highspeed region.

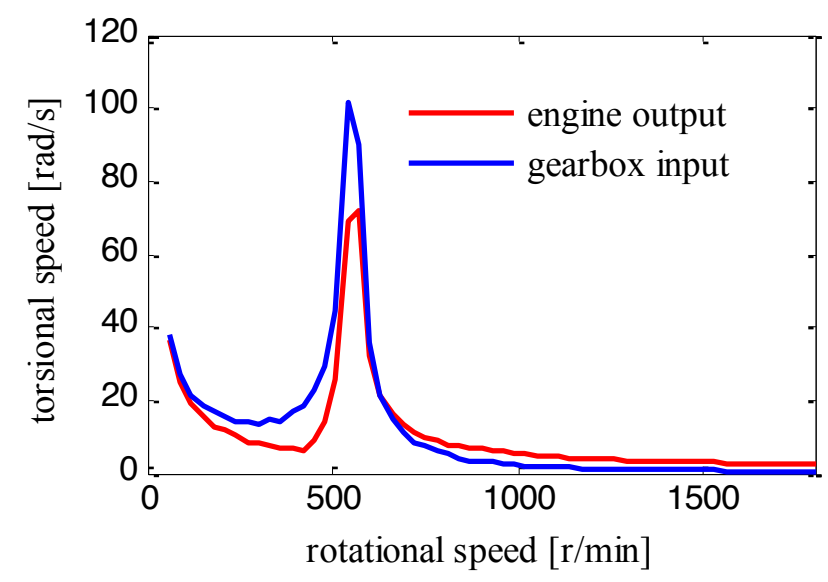

Fig. 16. Responses of the power transmission system

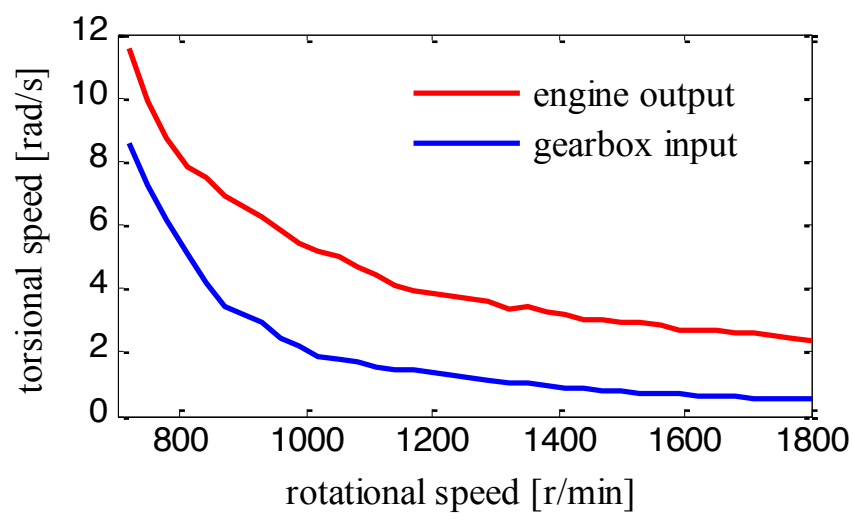

Fig. 17. Responses in high-speed region

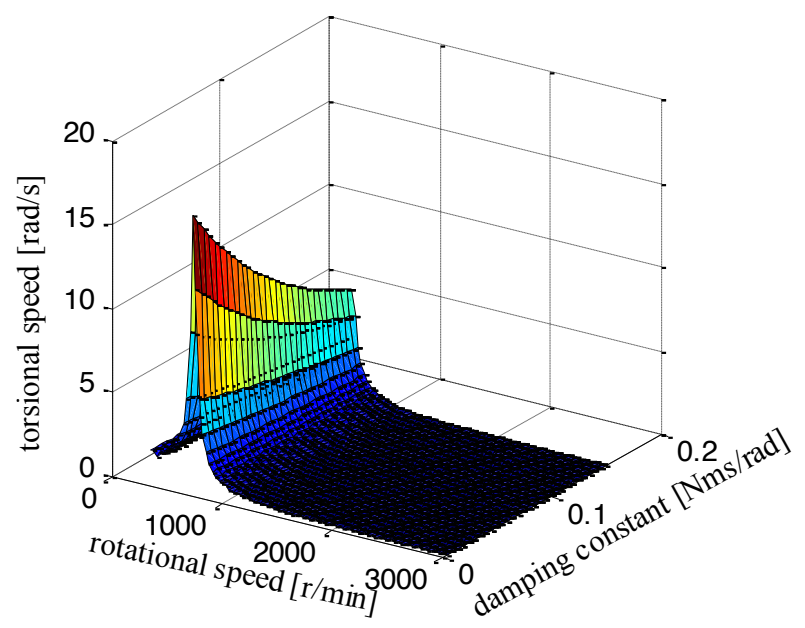

Fig. 18. Variations of system response and damping

\section{Conclusions}

To reveal the torsional vibration characteristics of the vehicle's power transmission system under full rotational speed, a hysteretic nonlinear model of the torsional damper was designed through experimental study and parameter estimation. The dynamic characteristics of torsional stiffness and damping of the torsional damper and the dynamic characteristics of the power transmission system were analyzed. The following conclusions are drawn:

(1) The elastic torque of the torsional damper can be described by the polynomial nonlinear function of the torsion angle. Parameters in the function are positively related to rotational speed. The equivalent torsional stiffness of the torsional damper increases as rotational speed increases.

(2) The damping torque of the torsional damper is described by the equivalent damping coefficient and damping factor. The damping factor increases as rotational speed increases. The proportion of viscous damping in the damping component and equivalent damping of the torsional damper also present the same changes.

(3) The resonant rotational speed of the power transmission system is lower than the idle speed (about $580 \mathrm{r} / \mathrm{min}$ ). Torsional stiffness and damping mainly influence the torsional vibration of the power transmission system in low-speed region. Since the torsional vibration amplitude of the system is lower as the rotational speed increases, torsional stiffness and damping of the torsional damper slightly improve the torsional vibration of the power transmission system under high-speed conditions.

In this study, a new understanding on the nonlinear characteristics of the torsional damper is proposed by combining experimental studies and theoretical modeling. The transmitted torque model of the torsional damper is conventional and can provide theoretical references for torsional vibration characteristic analysis and parameter optimization of the torsional damper under full rotational speed conditions. The modeling method is also applicable to other torsional dampers with different dynamic parameters. Driving conditions of vehicles are complicated. In this study, conclusions are applicable to modeling and analysis of the torsional damper based on the test bench. However, torsional vibration of internal combustion engines is not single-order but multi-order.. Studying the dynamic characteristics of torsional dampers under multi-order excitation can simulate real working conditions of vehicles more accurately.

\section{Acknowledgement}

This work was supported by the National Natural Science Foundation of China (Grant No. 51605182) and the Fundamental Research Funds for Central Universities (Grant No. 2662016QD001).

This is an Open Access article distributed under the terms of the Creative Commons Attribution License 


\section{References}

1. Walter, A., Kiencke, U., Jones, S., “Anti-Jerk \& idlespeed control with integrated sub-harmonic vibration compensation for vehicle with dual mass flywheel". SAE International Journal of Fuels and Lubricants, 1, 2009, pp.1267-1276.

2. Chen, T., Lv, Z. H., Su. C. Q., "Analysis Method for Elastic Characteristics of Arc Spring". China Mechanical Engineering, 17(5), 5, 2007, pp. 93-95

3. Ding, Y., Pan, Y. X., "Design and Research on Arc Spring in Dual Mass Flywheel". Journal of Wuhan University of Technology, 31(5), 2008, pp.114-117.

4. Huang, Z., Chen, D. M., Wang, D. J., Su, W., Guo, X. 1., "Design and Simulation Analysis on Arc Helix Spring of Dual Mass Flywheel". Journal of Academy of Armored Force Engineering, 23(4), 2009, pp.31-35.

5. Chen, L., Zeng, R., Jiang, Z. F., "Nonlinear dynamical model of an automotive dual mass flywheel". Advances in Mechanical Engineering, 7(6), 2015, pp.1-11.

6. Zhao, G. M., Jiang, Z. F., Chen. L., Zeng. R., "Research and Experimental Verification on Damping Characteristics of Circumferential Arc Spring Dual Mass Flywheel". Chinese Internal Combustion Engine Engineering, 33(1), 2012, pp.81-86

7. Li, Z., Sandhu, J., "Transmission Torque Converter Arc Spring Damper Dynamic Characteristics for Driveline Torsional Vibration Evaluation". SAE International Journal of Passenger Cars-Mechanical System, 6(1), 2013, pp.1483-1488.

8. Kukhyun, A., Jang, M. L., Wonsik, L., Yeong-il. P., "Analysis of a Clutch Damper Using a Discrete Model". KSME International Journal, 18(11), 2004, pp.1883-1890.

9. Schaper, U., Sawodny, O., Mahl. T., "Modeling and Torque Estimation of an Automotive Dual Mass Flywheel". In: 2009 American Control Conference, St. Louis, USA: IEEE, 2009, pp.1207-1212

10. Yao. L. D., Yang, H. N., Sun, H. W., Duan, Q. H., "Research of Rheology for the Lithium Grease". ACTA PETROLET SINICA (PETROLEUM PROCESSING SECTION), 10, 2011, pp. 1-5.

11. Shi, W. K., Long, Y., Lu Y. D., "Study on multistage non-linear dual mass flywheel damper". Journal of Vibration and Shock, 28(5), 2009, 92-96.

12. Lamkane, A.A., Kulkarni, S.N., Mali, V.V., "Design and Analysis of Dual Mass Flywheel Based on The Principle of Compensation and Continuously Variable Stiffness". International Journal of Application or Innovation in Engineering \& Management, 5(4), 2016, pp.124-131.

13. Lv, Z. H., Chen, T., "Design of the elastic characteristics of torsional damper with double mass flywheel and circumferential spring". Automotive Engineering, 28(1), 2006, pp.73-77.

14. Chen, L., Deng, M. R., Jiang, Z. F., "Optimization Method of Performance Parameters of Dual Mass Flywheel". Transactions of CSICE, 30(3), 2012, pp.277-283.

15. Shelke, R.S., Dighole, D.G., "A Review paper on Dual Mass Flywheel". International Journal of Science, Engineering and Technology Research. 5(1), 2016, pp.326-331.

16. Swapnil, L., Sonawane, D.P., Londhe, B.C., "Review on Design and Manufacturing of Dual Mass Flywheel". In: International Conference on Emerging Trends in Engineering and Management Research. Nashik, India: NGSPM's Brahma Valley College of Engineering \& Research Institute, 2016, pp.22-36
17. Yan, M. G., Hou, Z. C., Yang, F. Y., Yu, P., Ye, X., "Parameter Optimization on the Torsional Vibration Damper in the DriveLine of a Hybrid Electric Bus". Automobile Technology, 8, 2015, pp. 1-5.

18. Xiang, C. L., Li, H., Liu, H., "A Study on the Damping Characteristics of Semi-active Torsional Damper with Combined Positive and Negative Stiffness in Parallel". Automotive Engineering, 37(4), 2015, pp.430-434.

19. Philipp, M., Alexander, F., Arne K., Heiko G., "Simulation based optimization of torsional vibration dampers in automotive powertrains". Mechanism and Machine Theory, 115, 2017, pp. 244-266.

20. Yoon, J. Y., Kim, B., "Gear rattle analysis of a torsional system with multi-staged clutch damper in a manual transmission under the wide open throttle condition". Journal of Mechanical Science and Technology, 30(3), 2016, pp. 1003-1019.

21. Shu, G. Q., Wang, B., Liang, X. Y., "Torsional Vibration Reduction Analysis of Variable Damping Torsional Vibration Damper for Engine Crankshaft". Journal of Tianjin University(Science and Technology), 48(1), 2015, pp.19-24.

22. Tejashri, K., Patil, V.R., "Performance Evaluation of IC Engine Using Dual Mass Flywheel”. International Journal of Innovative Research in Science Engineering and Technology, 5(2), 2016, pp.1635-3645.

23. Shimizu, Y., Tsujiuchi1, N., Ito, A., Yamamoto, S., "Optimization of Flywheel Damper for Passenger Car to Reduce Acceleration/Deceleration Shock". In: International Conference on Noise and Vibration Engineering, KU Leuven, Belgium: ISMA, 2016, pp.3873-3882

24. Zheng, G. Z., Wang, C., Hao, T., "Influence of Dual Mass Flywheel on NVH Performance of Powertrains". Journal of Chongqing University of Technology (Natural Science), 32(5), 2018, pp.58-61.

25. Tejashri K., "Design Development and Comparative Analysis of Spring Mass Flywheel vs Conventional Flywheel for Two-stroke Engine". International Journal of IT, Engineering and Applied Sciences Research, 4(8), 2015, pp.1-11.

26. Liu, S. T., "Influences of a dual-mass flywheel damper on idling vibration". Transactions of the Chinese Society for Agricultural Machinery, 35(3), 2004, pp.16-19.

27. Song, L. Q., Zhao, X. F., He, Z. H., Luo, S. M., Tian, H. Y., Fan, Z. Z., "Analysis model and inherent characteristics of torsional vibration of the dual mass flywheel-circumferential short spring introduced friction". Journal of Mechanical Engineering, 45(11), 2009, pp.99-105.

28. Li, J., Yu, X. L., Zhang, P. W., Liu, H. J., "Torsional vibration test system based on virtual instrument". Journal of Mechanical \& Electrical Engineering, 28(12), 2011, pp.1430-1434.

29. Jiang, Z. F., Chen, L., Wu, B., Research on Method of Dual Mass Fly-wheel Dynamic Experimentation. Journal of Wuhan University of Technology, 30(10), 2008, pp. 117-119.

30. Hoon, W., Yoon, Y. K., Haeil, J., Gwang, N. L., "Nonlinear ratedependent stick-slip phenomena: modeling and parameter estimation". International Journal of Solids and Structures, 38, 2001, pp.1415-1431. 